\title{
IN-VITRO-EFFECTS OF TNF-A AND IL-1-ANTAGONISTS ON THE CYTOKINE NETWORK OF PATIENTS WITH OLIGOARTICULAR IDIOPATHIC ARTHRITIS (OJIA)
}

\author{
L. Strothmann, M. Kirchner, V.N. Umlauf, W. Mannhardt-Laakmann \\ Center for Pediatric and Adolescent Medicine, Pediatric Immunology and Rheumatology Johannes \\ Gutenberg-University of Mainz, Mainz, Germany
}

Background and aims: Pro-inflammatory cytokines are the main mediators of juvenile idiopathic arthritis. Modern therapeutic strategies (biologicals) are therefore geared to block the action of individual profinflammatory cytokines selectively. We investigated the effect of selective cytokine blocking on the equlilibrium of associated inflammatory mediators.

Methods: Lipopolysaccharid stimulation was performed in vitro on peripheral mononuclear cells from 10 patients with oJIA and 15 healthy individuals for 24 hours. Etanercept, Anakinra and Adalimumab were used to inhibit cytokine effects. In the collected culture supernatants the profile of cytokine production was studied by multiplex cytokine bead immunoassay.

Results: In healthy individuals selective cytokine inhibition prevented proinflammatory cytokine production collectively.In oJIA cell cultures with Adalimumab or Anakinra TNF- $\alpha$ or IL-1 $\beta$ concentrations were significantly decreased. In contrast this could not be shown for Etanercept (TNF- $\alpha$ ). We found decreased amounts of proinflammatory cytokines IL- $1 \beta$ and IFN- $\gamma$. A decrease of IL- 6 could be achieved only with Anakinra and Etanercept. Interestingly, after blockade of TNF- $\alpha$ or IL-1 $\beta$ we found increases in concentrations of proinflammatory IL-8 and anti-inflammatory IL-4.

Conclusions: Blockade of cytokine effects of TNF- $\alpha$ or IL-1 $\beta$ may cause the decline of further proinflammatory cytokines in oJIA patients. This selective anti-inflammatory effect is likely supported by an increase of IL-4. The simultaneous presence of proinflammatory IL-8 seems to account for the maintenance of autoinflammation. This could be an attempt to explain the pathogenesis of therapy-resistant disease progression in anti-cytokine therapy. Hence, the use of humanized antibodies against IL-8 may play a role in future treatment of JIA. 